Proceedings of the 43rd "Jaszowiec", International School and Conference on the Physics of Semiconductors, Wisła 2014

\title{
Optical and Structural Properties of GaAs/AlGaAs Quantum Wells Grown by MBE in the Vicinity of As-Rich-GaAs/ZnSe Heterovalent Interface
}

\author{
G.V. Klimko, E.A. Evropeytsev, A.A. Sitnikova, S.V. Gronin, I.V. Sedova, \\ S.V. SOROKIn AND S.V. IVANOV \\ Ioffe Physical-Technical Institute, Politekhnicheskaya 26, St. Petersburg 194021, Russia
}

\begin{abstract}
The studies of structural and optical properties of molecular beam epitaxy grown pseudomorphic hybrid structures with AlGaAs/GaAs quantum well placed closely to the GaAs/ZnSe heterointerface are presented. The interfaces were formed in different ways ( $\mathrm{n}$ or Se initial GaAs surface exposure, different growth temperature and ZnSe growth mode) on As-rich $\mathrm{c}(4 \times 4)$ and $(2 \times 4)$ GaAs surfaces. It has been demonstrated that the photoluminescence intensity from the near-heterointerface GaAs QW is influenced most significantly by the procedure of ZnSe growth initiation. The bright photoluminescence $(77 \mathrm{~K})$ from the near-interface GaAs quantum well is observed if the Se-decoration procedure is used during the GaAs/ZnSe heterointerface formation on $(2 \times 4)$ As GaAs surface. It reduces noticeably if the GaAs reconstruction changes to $\mathrm{c}(4 \times 4) \mathrm{As}$ and disappears completely when $\mathrm{Zn}$ pre-exposure of GaAs surface is used. These effects are discussed in terms of different ratio of Ga-Se and As-Zn bonds at the $\mathrm{GaAs} / \mathrm{ZnSe}$ heterointerface resulting in different band offsets and/or uncompensated built-in electric fields.
\end{abstract}

DOI: $10.12693 /$ APhysPolA.126.1184

PACS: 78.66.- $\mathrm{w}, 68.65 .-\mathrm{k}, 81.15 . \mathrm{Hi}$

\section{Introduction}

The molecular beam epitaxy (MBE) grown hybrid III-V/II-VI structures have attracted much attention in the last few years because of their prospects for optoelectronic applications and fundamental studies in spintronics. The existence of heterovalent interface (HI) near the active region extends significantly the opportunity of band engineering in semiconductor heterostructures, resulting in their improved functionality. In particular, mid-IR lasers based on hybrid Al$\mathrm{GaSb} / \mathrm{InAs} / \mathrm{ZnTe} / \mathrm{CdMgSe}$ heterostructures [1] as well as optically active heterovalent InAs quantum wells (QWs) [2] were demonstrated. In the field of spintronics the hybrid structure with a diamagnetic $\mathrm{AlGaAs} / \mathrm{GaAs}$ quantum well (QW) resonantly coupled to a paramagnetic $\mathrm{ZnSe} / \mathrm{ZnCdMnSe}$ QW through the III-V/II-VI HI has demonstrated the strong magnetic interaction [3], and a $60 \%$ spin injection from a $\mathrm{ZnMnSe}$ diluted magnetic semiconductor (DMS) injector to a double AlGaAs/GaAs QW placed in the vicinity of such HI has been observed [4].

Fabrication of the III-V/II-VI HI is the key point in the MBE growth of such hybrid structures. The HI band offsets are controlled by the interface atomic configuration and, consequently, by the III-V surface reconstruction and interface formation procedure $[5,6]$. Moreover, atomic intermixing across the HI implies some doping of the adjacent layers, and thus affects the carrier distribution and band bending around the interface $[7,8]$. The low density of extended defects at HI is also of a great importance if the $\mathrm{HI}$ is located in the vicinity of the active layer(s) of the structures. The above mentioned hybrid structures $[3,4]$ contained the thermodynamically equilibrium neutral GaAs/ZnSe HI with chemical band offsets $\left(\Delta E_{\mathrm{C}} \approx 170 \mathrm{meV}\right)[3]$ correlating well with the theoretically predicted value [6]. It was formed using high temperature $\left(T_{\mathrm{S}}=300^{\circ} \mathrm{C}\right) \mathrm{ZnSe} \mathrm{MBE}$ deposition on $(2 \times 4)$ As GaAs surface mediated by a controllable Se-decoration yielding a $(2 \times 1)$ Se reconstruction [9].

The studies of III-V/II-VI HI formation on $\mathrm{c}(4 \times 4) \mathrm{As}$ $\mathrm{GaAs}$ is of a great importance for Be-containing hybrid structures, e.g. $p^{+}$-GaAs $/ n^{+}$-ZnSe tunneling diodes in hybrid multi-junction solar cells, because of the low growth temperature $T_{\mathrm{S}}=400-450{ }^{\circ} \mathrm{C}$ necessary for highly p-doped $(\mathrm{Al}) \mathrm{GaAs}$ :Be to suppress fast Be diffusion and segregation [10]. Low $T_{\mathrm{S}}$ results in the $\mathrm{c}(4 \times 4) \mathrm{As}$ reconstruction of the as-grown GaAs structure.

This paper presents the results of comparative studies of structural and optical properties of hybrid structures with the $\mathrm{AlGaAs} / \mathrm{GaAs}$ QW placed closely to the GaAs/ZnSe HI formed in different ways on As-rich $c(4 \times 4)$ and $(2 \times 4)$ GaAs surfaces.

\section{Experiment}

The (Al)GaAs/ZnSe hybrid structures were grown on semi-insulating $\mathrm{GaAs}(100)$ substrates using a doublechamber MBE setup (SemiTEq, Russia). They contain two GaAs QWs with thicknesses of $10 \mathrm{~nm}$ and $6 \mathrm{~nm}$ (top), separated by a $40 \mathrm{~nm}$ thick $\mathrm{Al}_{0.35} \mathrm{Ga}_{0.65} \mathrm{As}$ barrier. The top barrier comprises $3.5 \mathrm{~nm}$ thick AlGaAs capped with one monolayer $(\approx 0.3 \mathrm{~nm})$ of GaAs. In addition to the $\mathrm{c}(4 \times 4)$ As GaAs terminating reconstruction (structures $B, E, F)$, the $(2 \times 4)$ As one (structures $A$, $C, D)$ was also used as a reference. The III-V surface reconstructions were preserved during transfer to the IIVI growth chamber through ultrahigh vacuum (UHV). The variable parameters in the HI formation procedure 
were: the element of initial GaAs surface exposure ( $\mathrm{Zn}$ or Se), the growth temperature, and ZnSe growth mode (MBE or migration enhanced epitaxy (MEE)). The II-VI growth in structures $A$ and $B$ was initiated via the $30 \mathrm{~s}$ Zn pre-exposure followed by the ZnSe deposition in lowtemperature $\left(T_{\mathrm{S}} \approx 210^{\circ} \mathrm{C}\right) \mathrm{MEE}$ growth mode. In case of $(2 \times 4) \mathrm{As}$ GaAs surface, this is a "standard" GaAs/ZnSe HI formation procedure used for fabrication of II-VI laser structures, characterized by a low density of stacking faults nucleated at HI $[11,12]$. In structures $C, D, E, F$ the $\mathrm{ZnSe}$ growth was initiated on the GaAs surface preliminary decorated by Se background flux (with the main shutter closed $)$ until the appearance of clear $(2 \times 1)$ Se reconstruction [9]. Thereafter the ZnSe growth proceeded either in MBE (structures $D, F$ ) and MEE (structures $C$, $E$ ) modes at $T_{\mathrm{S}} \approx 280^{\circ} \mathrm{C}$. The growth parameters of the structures are outlined in Table. The reflection high energy electron diffraction (RHEED) was used for in situ control of III-V/II-VI HI formation. Structural properties were characterized by a transmission electron microscopy (TEM) in cross-section geometry (Philips EM420 microscope, $100 \mathrm{kV}$ ). Photoluminescence (PL) measurements were carried out at $T=77 \mathrm{~K}$, and the Cube $(\lambda=440 \mathrm{~nm})$ laser was used as an excitation source.

\section{TABLE}

The growth parameters of the structures. \#1 - Structure, \#2 - GaAs surface reconstruction, \#3 - Initial GaAs surface treatment, \#4 - ZnSe initial growth mode (temperature $\left.\left[{ }^{\circ} \mathrm{C}\right]\right), \# 5$ - Growth temperature of AlGaAs/GaAs QW $\left[{ }^{\circ} \mathrm{C}\right], \# 6$ - Defect density $\left[\mathrm{cm}^{-2}\right]$ (TEM).

\begin{tabular}{c|c|c|c|c|c}
\hline \hline$\# 1$ & $\# 2$ & $\# 3$ & $\# 4$ & $\# 5$ & $\# 6$ \\
\hline$A$ & $(2 \times 4) \mathrm{As}$ & Zn pre-exposure & MEE $(210)$ & 580 & $<10^{4}[13]$ \\
$B$ & $\mathrm{c}(4 \times 4) \mathrm{As}$ & Zn pre-exposure & $\operatorname{MEE~}(210)$ & 420 & $>10^{8}$ \\
$C$ & $(2 \times 4) \mathrm{As}$ & Se decoration & $\operatorname{MEE~}(280)$ & 580 & $<10^{6}$ \\
$D$ & $(2 \times 4) \mathrm{As}$ & Se decoration & $\operatorname{MBE}(280)$ & 580 & $<10^{6}$ \\
$E$ & $\mathrm{c}(4 \times 4) \mathrm{As}$ & Se decoration & $\operatorname{MEE~}(280)$ & 420 & $<10^{6}$ \\
$F$ & $\mathrm{c}(4 \times 4) \mathrm{As}$ & Se decoration & $\mathrm{MBE}(280)$ & 580 & $<10^{6}$
\end{tabular}

\section{Results and discussion}

Continuous RHEED monitoring demonstrated absence of $3 \mathrm{D}$ growth mode at the II-VI initial growth stage for all the above cases, which evidences formation of coherent interface. The detailed information on the HI structural quality, which confirmed the RHEED observation, was provided by TEM studies. Figure 1 presents a typical cross-sectional TEM image of the hybrid heterostructure (sample $F$ ). The two GaAs QWs as well as the III-V/II-VI HI are clearly resolved in the image. No misfit dislocations as well as stacking faults (SFs) were observed in all samples (except for sample $B$ ) within the resolution limit of cross-sectional TEM, being at the level of about $10^{6} \mathrm{~cm}^{-2}$. The SFs density in sample $B$ possessing a standard "laser" Zn-MEE growth initiation on $c(4 \times 4)$ As GaAs surface is above $10^{8} \mathrm{~cm}^{-2}$, which is in good agreement with results of Ref. [13]. The upper limits of extended defect densities for different HI are shown in Table.

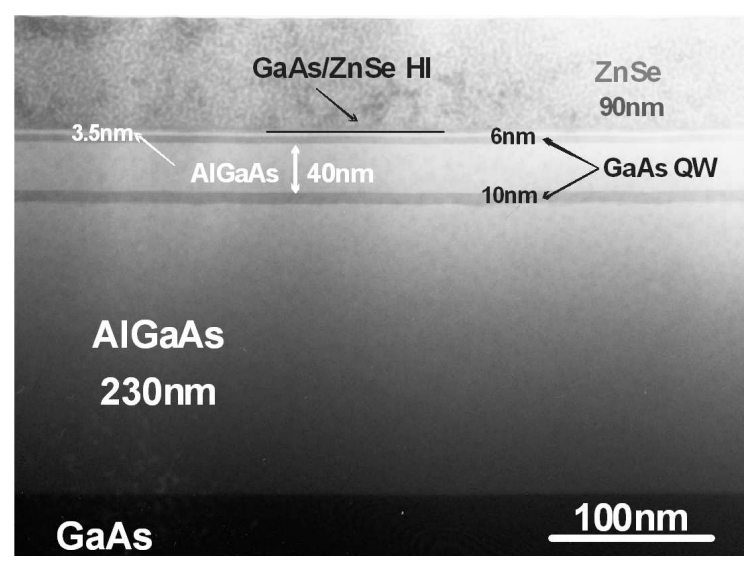

Fig. 1. Cross-sectional TEM image of the sample $F$.

The results of PL studies depend on the HI formation procedure. The most significant effect relates to the $\mathrm{ZnSe}$ growth initiation rather than the initial GaAs surface reconstruction $((2 \times 4)$ As or $c(4 \times 4)$ As $)$. No luminescence from the near-interface $6 \mathrm{~nm}$ thick GaAs QW was observed in samples $A$ and $B$ where HI was formed using the $\mathrm{Zn}$ pre-exposure and ZnSe deposition in low-temperature MEE mode. However, the PL intensity of the bottom $10 \mathrm{~nm}$ thick QW located relatively far $(\approx 50 \mathrm{~nm})$ from HI does not change much in these samples, as compared to reference pure III-V AlGaAs/GaAs QWs.

Contrary to that, the PL spectra of all other structures $(C, D, E, F)$ (Fig. 2) with Se decoration procedure used in the GaAs/ZnSe HI formation display pronounced emission from both GaAs QWs. The PL intensity from the near-HI QW dominates the spectra for samples $C$ and $D$ grown on $(2 \times 4) \mathrm{As}$ GaAs reconstruction, whereas in samples $E$ and $F(c(4 \times 4) A s)$ it is noticeably lower as compared with the emission intensity from the wide bottom QW, independently of the ZnSe growth mode (MEE or MBE). The bottom QW in all samples demonstrates approximately the same PL intensity within the $10 \%$ fluctuation range. From comparison of PL spectra of samples $E$ and $F$ having the same GaAs surface reconstruction but different growth temperatures of AlGaAs QWs (420 and $580^{\circ} \mathrm{C}$, respectively) one can conclude that it is the HI structure that governs the PL intensity of the nearinterface QW at least at $77 \mathrm{~K}$. Finally, MEE growth of $\mathrm{ZnSe}$ at $T_{\mathrm{S}} \approx 280^{\circ} \mathrm{C}$ on $(2 \times 4) \mathrm{As}$-Se-decorated GaAs surface has been found to provide the highest PL intensity from top GaAs QW in comparison with MBE one (sample $C$ vs. $D$ ).

Based on these findings one can confirm the importance of mixing of $\mathrm{As}-\mathrm{Zn}$ or $\mathrm{Ga}-\mathrm{Se}$ bonds at the HI, which leads to minimization of both the local electric fields and the interface charge, making the HI thermodynamically equilibrium with $\Delta E_{\mathrm{C}} \approx 150 \mathrm{eV}$ [6]. Most efficiently it is realized when the $(2 \times 4) \mathrm{GaAs}$ surface with an $\approx 75 \%$ As coverage catches Se atoms which form $\mathrm{Ga}-$ Se bonds in addition to As-Zn ones occurred during the ZnSe growth initiation (samples $C$ and $D$ ). This provides 


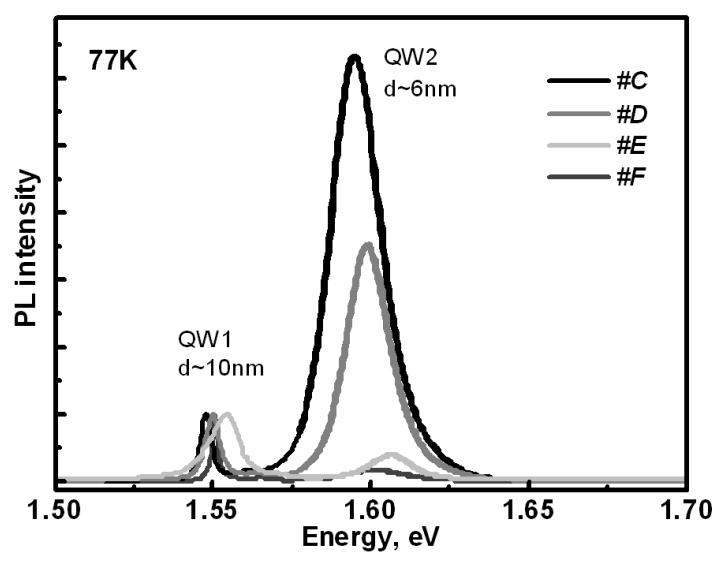

Fig. 2. PL spectra of samples $C, D, E, F$ at $77 \mathrm{~K}$, normalized to the emission intensity of the bottom GaAs QW.

good enough electron confinement in the top GaAs QW having otherwise a thin $(3.5 \mathrm{~nm})$ leaky barrier and minimizes electrostatic effect of HI. This result agrees well with our previous conclusions [3] and experimental results of Nicolini et al. [5]. The change of GaAs surface reconstruction to more As-rich $\mathrm{c}(4 \times 4)$ having the extramonolayer As coverage (samples $E$ and $F$ ) makes the Se decoration procedure less efficient in formation of $\mathrm{Ga}$ Se bonds which may either reduce the GaAs/ZnSe $\Delta E_{\mathrm{C}}$ value in accordance with [5] or enhance the electric field at the HI. Both these effects result in reduction of the $\mathrm{PL}$ intensity from top QW due to worse confinement or carrier depletion, respectively. Finally, the $\mathrm{Zn}$ pre-exposure seems to make the HI possessing preferably the As-Zn bonds independently of the GaAs surface reconstruction, which further reduces $\Delta E_{\mathrm{C}}[5]$ and increases the HI field. As a result no PL from the near-interface GaAs QW is observed in samples $A$ and $B$. Of course, one should not neglect a possible effect of GaAs surface reconstruction and ZnSe growth initiation procedure on the inter-diffusion of group II and VI elements into near-interface III-V QW, which may also influence its PL intensity. However this assumption should be checked by detailed secondary ion mass-spectroscopy and X-ray photoelectron spectroscopy studies which are under the progress now.

\section{Conclusions}

The studies of structural and optical properties of hybrid structures with the AlGaAs/GaAs QW placed closely to the GaAs/ZnSe HI formed in different ways on As-rich $\mathrm{c}(4 \times 4)$ and $(2 \times 4)$ GaAs surfaces were carried out. It has been demonstrated that the $\mathrm{ZnSe}$ growth initiation has the most significant effect on PL intensity from the near-interface GaAs QW. No luminescence has been observed for the case of HI formation using the $\mathrm{Zn}$ pre-exposure and $\mathrm{ZnSe}$ deposition in lowtemperature MEE mode, whereas the samples with Se decoration of the initial GaAs surface have displayed pronounced emission from both GaAs QWs. The PL intensity from the near-HI QW in case of $\mathrm{c}(4 \times 4) \mathrm{As}-\mathrm{GaAs} / \mathrm{Se}-$
decoration/ZnSe HIs has been revealed to be noticeably lower as compared with that at the $(2 \times 4) \mathrm{As}-\mathrm{GaAs} / \mathrm{Se}-$ decoration/ZnSe HIs presumably due to lower density of Ga-Se bonds at the HI, which reduces GaAs/ZnSe $\Delta E_{\mathrm{C}}$ and/or increases uncompensated electric fields at the HI. All the structures demonstrate the SFs density nucleated at the $\mathrm{HI}$ at the level well below $10^{6} \mathrm{~cm}^{-2}$, except for the case of $\mathrm{Zn}$-exposure growth initiation on the $c(4 \times 4) \mathrm{As}$ GaAs surface $\left(10^{8} \mathrm{~cm}^{-2}\right)$.

\section{Acknowledgments}

The work was partly supported by RFBR grant \#1302-01063.

\section{References}

[1] S.V. Ivanov, V.A. Kaygorodov, S.V. Sorokin, B.Ya. Meltser, V.A. Solov'ev, Ya.V. Terent'ev, O.G. Lyublinskaya, K.D. Moiseev, E.A. Grebenshchikova, M.P. Mikhailova, A.A. Toropov, Yu.P. Yakovlev, P.S. Kop'ev, Zh.I. Alferov, Appl. Phys. Lett. 82, 3782 (2003).

[2] S.V. Ivanov, O.G. Lyublinskaya, Yu.B. Vasilyev, V.A. Kaygorodov, S.V. Sorokin, I.V. Sedova, V.A. Solov'ev, B.Ya. Meltser, A.A. Sitnikova, T.V. L'vova, V.L. Berkovits, A.A. Toropov, P.S. Kop'ev, Appl. Phys. Lett. 84, 4777 (2004).

[3] A.A. Toropov, I.V. Sedova, S.V. Sorokin, Ya.V. Terent'ev, E.L. Ivchenko, S.V. Ivanov, Phys. Rev. B 71, 195312 (2005).

[4] F. Liaci, V.Kh. Kaibyshev, A.A. Toropov, Ya.V. Terent'ev, M.S. Mukhin, G.V. Klimko, S.V. Gronin, I.V. Sedova, S.V. Sorokin, S.V. Ivanov, Phys. Status Solidi C 9, 1790 (2012).

[5] R. Nicolini, L. Vanzetti, G. Mula, G. Bratina, L. Sorba, A. Franciosi, M. Peressi, S. Baroni, R. Resta, A. Baldereschi, J.E. Angelo, W.W. Gerberich, Phys. Rev. Lett. 72, 294 (1994).

[6] A. Kley, J. Neugebauer, Phys. Rev. B 50, 8616 (1994).

[7] A. Colli, E. Carlino, E. Pelucchi, V. Grillo, A. Franciosi, J. Appl. Phys. 96, 2592 (2004).

[8] A. Frey, U. Bass, S. Mahapatra, C. Schumacher, J. Geurts, K. Brunner, Phys. Rev. B 82, 195318 (2010).

[9] I.V. Sedova, G.V. Klimko, S.V. Sorokin, V.K. Kaibyshev, Ya.V. Terentyev, A.A. Toropov, S.V. Ivanov, in: Abstr. 17th Int. Conf. on Molecular Beam Epitaxy (MBE 2012), Nara (Japan), 2012, p. 251.

[10] S.V. Ivanov, P.S. Kop'ev, N.N. Ledentsov, J. Cryst. Growth 108, 661 (1991).

[11] E. Kato, H. Noguchi, M. Nagai, H. Okuyama, S. Kijima, A. Ishibashi, Electron. Lett. 34, 282 (1998).

[12] S. Miwa, L.H. Kuo, K. Kimura, T. Yasuda, A. Ohtake, C.G. Jin, T. Yao, Appl. Phys. Lett. 73, 939 (1998).

[13] S.V. Gronin, I.V. Sedova, S.V. Sorokin, G.V. Klimko, K.G. Belyaev, A.V. Lebedev, A.A. Sitnikova, A.A. Toropov, S.V. Ivanov, Phys. Status Solidi $C$ 9, 1833 (2012).

[14] L.H. Kuo, K. Kimura, T. Yasuda, S. Miwa, C.G. Jin, K. Tanaka, T. Yao, Appl. Phys. Lett. 68(17), 2413 (1996). 